\title{
FORECASTING OF THE PRODUCTIVITY OF PARTS MACHINING BY HIGH- SPEED MILLING WITH THE METHOD OF HALF-OVERLAP
}

\author{
Sergey DOBROTVORSKIY ${ }^{1}$, Yevheniia BASOVA ${ }^{2}$, Maryna IVANOVA ${ }^{3}$, \\ Alexey KOTLIAR ${ }^{4}$, Ludmila DOBROVOLSKA ${ }^{5}$ \\ National Technical University "Kharkov Polytechnic Institute", Department of Technology of Mechanical \\ Engineering and Metal-Cutting Machine Tools, 2, Kyrpychova str., 61002, Kharkov, Ukraine \\ e-mail: sdobro50@gmail.com; ${ }^{2}$ e-mail: e.v.basova.khpi@gmail.com; \\ 3e-mail: ivanovamaryna1@gmail.com, ${ }^{4}$ e-mail: Alexeykotliar@gmail.com, ${ }^{5}$ e-mail: lyudmyla@ukr.net
}

Abstract

In the article the technology of high-speed milling was observed as a key to the most promising methods of engineering products machining. It is indicated that the correct choice of the strategy for moving the cutting tool in the manufacture of products is the basis for ensuring the desired surface quality of the workpiece and its high processing efficiency. A review of the work devoted to the diagnosis of high-speed processing strategies was performed. Based on the up-to-date knowledge of the physics of the process of establishing of a qualitative surface, the article proposes to consider a new technology of high-speed milling with half-overlap. As the main idea of this technology, it is suggested to consider the work of the deformation component of cutting process as the main component of the complex energy-intensive process of high-speed milling. In addition, the paper suggests a technique for estimating the productivity of machining by the method of high-speed milling with half-overlap. It is theoretically justified that the specific productivity of the proposed technology is quite high $\mathrm{Q}=12.5 \div 4.2 \mathrm{~cm}^{2} / \mathrm{min}$ and depends on the technological regimes. The place of technology of high-speed milling with half-overlap in the technological process of manufacturing details of any complexity was noted. The area of further research is indicated.

Keywords: high-speed milling, processing efficiency, processing strategy, half-overlap, deformation component.

\section{INTRODUCTION}

An increasing of quality requirements of engineering products is one of the reasons for the development of metal processing techniques. Currently, the manufacturing process of parts machining can be optimally unified and the most adapted to the capabilities of modern metal-cutting equipments and tools, using the knowledge of the physics of forming a high-quality surface.

From the analysis of the most using technologies for manufacturing irregular shape workpiece or critical parts of machines, it is known that one of the most future-oriented technologies for shaping with the desired characteristics of the machined surface quality is the technology of highspeed milling at modern multi-axis machining centers. However, the problem of implementing high-speed milling (HSM) technology consists is not only in the selection of optimal machining conditions, which are critically different for each material, but also in the appropriation of the tool path strategy for HSM.

The choice of the optimal tool path strategy of high-speed milling at the finishing steps of the products manufacturing technological process will make possible increasing of high quality and accuracy of the any complexity surface and will exclude abrasive technological operations from the manufacturing process. Therefore, the actual modern task is to find technological solutions for the development of alternative finishes surface technologies that can contribute high machined part quality indicators.

The central purpose of the presented work is to establishes the applicability and forecasting of productivity of high-speed milling technology with method of half-overlap using ball-end milling tools in the machine parts manufacturing.

\section{ANALYSIS OF INFORMATION SOURCES}

There are many components to an effective high speed machining process for critical machinery parts. A large number of works has been written about the impact HSM has had on CNC machine tools, spindles, toolholders, cutting tools, and controls.

It is known that in the context of high-speed milling operations, the tool path strategy has a significant influence on the cycle time. This means that, for the removal of the same amount of material, the type of tool motion utilized will produce significantly different results in terms of cycle time [1].

In work [1] at look discusses the influence of the tool path strategy on the cycle time of highspeed milling operations. The essence of the 
proposed method is based on the experimental measurement of the machine tool acceleration and specific geometric assumptions regarding tool motion. In work [2] an objective function concerning machining cost and associated optimization algorithm based on machining time and cutting length calculation was proposed.

Over the last few years, new strategies for highspeed roughing have been introduced that offer significant increases in metal removal rates and provide much shorter machining times [3-7]. In paper [8], six different tool path strategies have been investigated on the surface finish and machining time of a rectangular cavities of ESR Stavax material. Here, the authors noted that in order to effectively use the HSM capabilities, optimizing the tool path strategies and machining parameters is an important issue.

In paper [9] author group have elaborated an optimization method based on the analysis of the different critical parameters so as to distinguish the most suitable strategies to calculate the cutting time and define the machine dynamics.

Thus, the question of determining the optimal cutting strategy for surface parts is an important task in modern machine building. Analysing the modern employed paths of tool movements in HSM, it was determined that information about using of technology with half-overlap to date in the sources of information are not available but the technology itself may be of particular interesting at the final technological process step of the machines part manufacturing.

\section{SUBSTANTIATION OF THE POSSIBILITY FOR USING HIGH-SPEED PROCESSING TECHNOLOGY WITH HALF-OVERLAP}

At milling by ball end mill cutter of size in the longitudinal and transverse directions surface roughness are widely divergent. The value of the transverse roughness $\left(\mathrm{Ra}_{\text {trans }}\right)$ may exceed the value of the longitudinal roughness $\left(\mathrm{Ra}_{\text {long }}\right)$ by several times, which is explained by the presence of residual scallops between adjacent cutter passes. Increasing the speed of processing, compared with the traditional milling technology, suggests that when choosing an adequate strategy, it is possible to achieve such a mechanism for the formation of transverse and longitudinal roughness, which corresponds to the result characterizing the surface after grinding.

If the allegations made was confirmed, the surface roughness can be considered in the limit as a white noise signal, which consists of periodic, random components and their product (superposition) [10]. Such an approach to solving the problem can be realized using the inverse Laplace transform (1), and to make the right decision - the Pearson criterion $-\mathrm{x}^{2}(2)$ :

$$
f(x)=L^{-1}\{F(s)\}=\frac{1}{2 \pi i} \int_{\sigma_{1}-i \cdot \infty}^{\sigma_{1}+i \cdot \infty} e^{s x} F(s) d s,
$$

where

$f(x)$ - real variable (the original of the processed surface);

$\sigma_{1}$ - some real number;

$\mathrm{F}(\mathrm{s})$ - complex variable (the image of the processed surface);

$$
x^{2}=N \sum \frac{\left(P_{i}^{\text {theor }}-P_{i}^{\text {emp }}\right)^{2}}{P_{i}^{\text {theor }}},
$$

where $P_{i}^{\text {theor }}=\int_{x_{i-1}}^{x_{i}} f(x) d x$ - likelihood of falling into the i-th interval; $\quad P_{i}^{e m p}=\frac{n_{i}}{N}-\quad$ corresponding empirical value,

$\mathrm{n}_{\mathrm{i}}$ - the number of sampling elements from the $\mathrm{i}$ th interval.

However, the development of this approach is possible with the creation of a finishing technology with the maximum release of the deformation component of the cutting process. Because in the energy balance of the cutting process, $90 \%$ or more cutting energy has been accounted for by work of plastic deformation of the material of the workpiece. From the plastic deformation depends on the thermal regime and contact loads on the working surfaces of the tool, and, consequently, the intensity and of the tool wear patterns. The quality of the resulting surface depends on the conditions of plastic deformation and the mechanism of strain hardening. The value of the deformation component depends on the hardness of the material being processed.

Thus, the main idea of the work being presented is to create a technology that contains a mechanic working method that makes it possible to strengthen the role of deformation processes. This method we call as the method of high-speed milling with a half-overlap. The essence of the method lies in the fact that after performing the linear processing we're processing the surface with an equidistant trajectory with a half step along the vertices of the previously obtained scallops (fig. 1) [11].

Consequently, provides an opportunity not only to directly cut off the tops of the scallops, but also to deform them. Thus, the potential of high-speed milling technology (the deformation component of the cutting process) is released in the process of forming the quality of the processed surface. This in turn has allowed us to assume that the roughness after such a machining method will be low, and there will be no unfavorable transformations in the surface layer of the material, as, for example, when grinding.

The problem that arises of the appliance of this method in comparison with traditional line processing is process efficiency. 


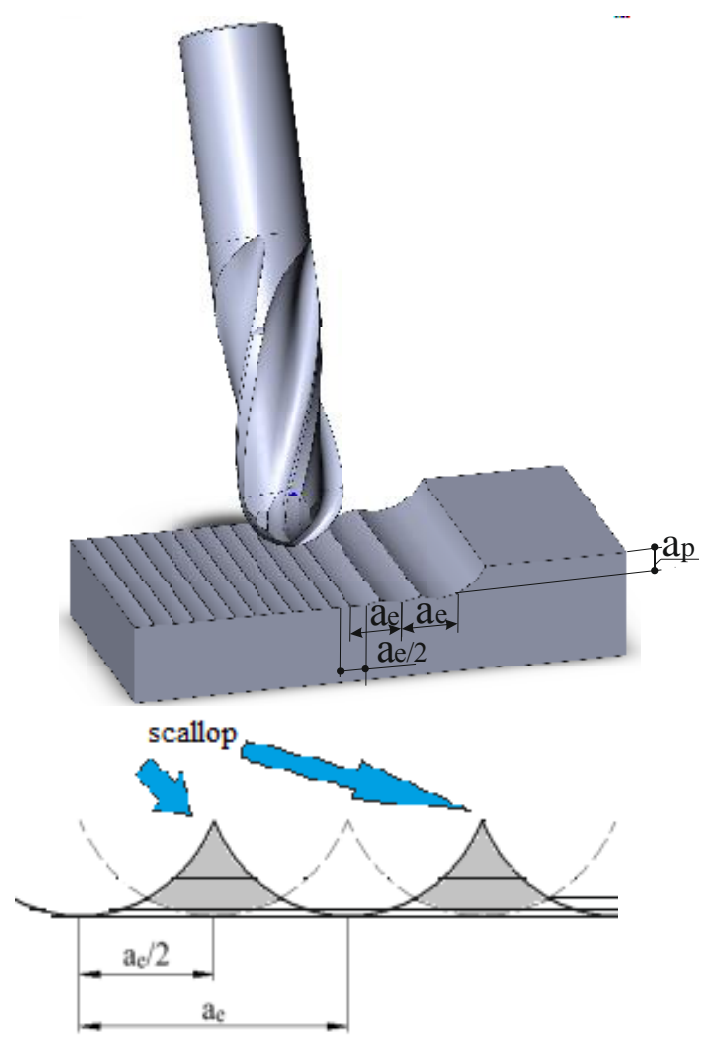

Fig. 1. Chart of implementing high-speed milling technology by linear processing with half-overlap: $\mathrm{a}_{\mathrm{e}}$ - step between adjacent tool passes; $a_{\mathrm{e}} / 2$ - half-overlap value

\section{EFFICIENCY EVALUATIONS OF THE TECHNOLOGY OF LINE HIGH-SPEED MILLING WITH A HALF-OVERLAP}

To implement an analysis of the productivity of the high-speed milling technology with halfoverlap, we developed the following methodology

$$
\begin{aligned}
& L=L_{1} \cdot n_{1}+L_{1} \cdot(n-1)+L_{2} \cdot 2 n_{1}+ \\
& +L_{3} \cdot 2 n_{1}+L_{4} \cdot 2 n_{1}+L_{5} \cdot n_{2}
\end{aligned}
$$

where

$\mathrm{L}$ - the total length of the road of the cutting tool;

$\mathrm{L}_{1}$ - the total length of the one pass of the cutting tool;

$\mathrm{n}_{1}$ - number of passes;

$\mathrm{L}_{2}$ - the total length of the transverse pass;

$\mathrm{L}_{3}$ - the total length of milling by air;

$\mathrm{L}_{4}$ - the total length of the rapid motion of the cutting tool;

$\mathrm{L}_{5}$ - fast supply and discharge of the cutting tool into points of half-overlap;

$\mathrm{n}_{2}$ - number of supply and discharge of the cutting tool (by working with one tool $\mathrm{n}_{2}=2$ ).

Based on the theory given in the literature [1215] determine the length of the path of the cutting tool during machining:

$$
L=\int_{0}^{t} \sqrt{\Omega^{\prime T} \cdot M_{p} \cdot \Omega^{\prime}} \frac{d \lambda}{d t},
$$

where
$\Omega^{\text {T }}$ - is consistent with the matrix $\left\lceil\frac{\partial u}{\partial \lambda} \frac{\partial v}{\partial \lambda}\right\rceil$,

that corresponds to $C(\lambda)$ based in the field of coordinate (u, v) (Fig. 2);

$\mathrm{t}$ - value of the time component in the process of cutting, sec;

$\mathrm{M}_{\mathrm{p}}$ - the first defining surface matrix

$$
M_{p}=\left[\begin{array}{ll}
\frac{\partial P}{\partial u} \cdot \frac{\partial P}{\partial u} & \frac{\partial P}{\partial u} \cdot \frac{\partial P}{\partial v} \\
\frac{\partial P}{\partial v} \cdot \frac{\partial P}{\partial u} & \frac{\partial P}{\partial v} \cdot \frac{\partial P}{\partial v}
\end{array}\right]
$$

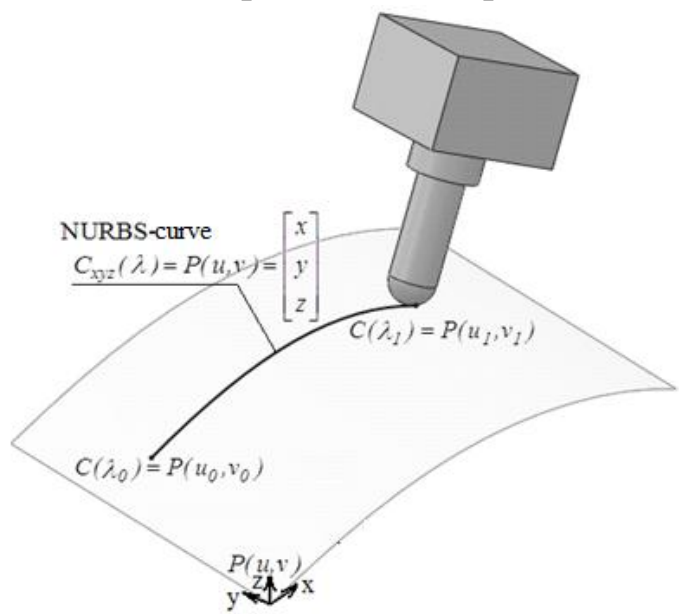

Fig. 2. Representing the NURBS curve on the NURBS surface

When considering the milling time, without taking into account the idle moves of the tool, the equation (1) for the efficiency evaluations of the technology of line high-speed milling with a halfoverlap of the complex surface (Fig.3) (NURBSsurface) has the view:

$$
\begin{aligned}
& L \approx L_{1} \cdot n_{1}+L_{1} \cdot(n-1)= \\
& =n_{1} \int \sqrt{\Omega^{\prime T} \cdot M_{p} \cdot \Omega^{\prime}} \frac{d \lambda}{d t}+ \\
& +(n-1) \int \sqrt{\Omega^{\prime T} \cdot M_{p} \cdot \Omega^{\prime}} \frac{d \lambda}{d t}
\end{aligned}
$$

or

$$
\begin{aligned}
& L=n_{1} \cdot \int_{0}^{t} \sqrt[\left[\begin{array}{ll}
\frac{\partial u}{\partial \lambda} & \frac{\partial v}{\partial \lambda}
\end{array}\right] \cdot\left[\begin{array}{lll}
\frac{\partial P}{\partial u} \cdot \frac{\partial P}{\partial u} & \frac{\partial P}{\partial u} \cdot \frac{\partial P}{\partial v} \\
\frac{\partial P}{\partial v} \cdot \frac{\partial P}{\partial u} & \frac{\partial P}{\partial v} \cdot \frac{\partial P}{\partial v}
\end{array}\right] \cdot\left[\begin{array}{l}
\frac{\partial u}{\partial \lambda} \\
\frac{\partial v}{\partial \lambda}
\end{array}\right]]{\frac{d \lambda}{d t}+} . \\
& +\left(n_{1}-1\right) \cdot \int_{0}^{t}\left[\begin{array}{ll}
\frac{\partial u}{\partial \lambda} & \frac{\partial v}{\partial \lambda}
\end{array}\right] \cdot\left[\begin{array}{lll}
\frac{\partial P}{\partial u} \cdot \frac{\partial P}{\partial u} & \frac{\partial P}{\partial u} \cdot \frac{\partial P}{\partial v} \\
\frac{\partial P}{\partial v} \cdot \frac{\partial P}{\partial u} & \frac{\partial P}{\partial v} \cdot \frac{\partial P}{\partial v}
\end{array}\right] \cdot\left[\begin{array}{l}
\frac{\partial u}{\partial \lambda} \\
\frac{\partial v}{\partial \lambda}
\end{array}\right] \frac{d \lambda}{d t}
\end{aligned}
$$

This efficiency evaluations of the technology is acceptable for NURBS interpolation for tool movement along a curve [12], which is used, for example, in G-code "G6.2". That command expands the choices from traditional linear and circular interpolation to interpolation along a spline represented by control points and knot points [4]. 


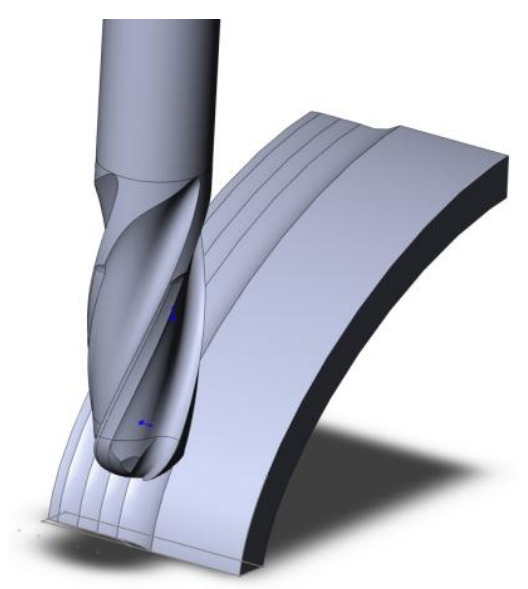

Fig. 3. Chart of processing a complex surface using high-speed milling technology with a half-overlap

To evaluate the machining of a plane surface, equation (1) acquires of the view

$$
\begin{aligned}
& L=L_{1} \cdot n_{1}+L_{1} \cdot(n-1)+L_{2} \cdot 2 n_{1}+L_{3} \cdot 2 n_{1} \approx \\
& \approx L_{1} \cdot 2 n_{1}+L_{2} \cdot 2 n_{1}+L_{3} \cdot 2 n_{1}
\end{aligned}
$$

To assess the adequacy of the mathematical expressions obtained, we calculated the productivity of the high-speed milling technology with half-overlap for processing a surface of 25 $\mathrm{cm}^{2}$.

To determine the processing time, the formula 4 was transformed into the following view:

$$
t=\frac{2 L_{1} \cdot n_{1}}{S}+\frac{2 L_{2} \cdot n_{1}}{S_{2}}+\frac{2 L_{3} \cdot n_{1}}{S x \cdot x},
$$

where

$\mathrm{t}$ - total working time of the cutting tool, sec;

$\mathrm{S}, \mathrm{S}_{2}$ - feed the tool during processing, $\mathrm{mm} / \mathrm{min}$;

$\mathrm{S}_{\mathrm{xx}}$ - feed the tool at idling speed, $\mathrm{mm} / \mathrm{min}$.

It was established that high-speed milling with half-overlap has a rather high productivity (Fig. 4). The results of a theoretical evaluation of the productivity of high-speed milling with a half- overlap when processing a flat surface of $25 \mathrm{~cm}^{2}$ are shown in tabl. 1.

Table 1. Productivity evaluations of the technology of

\begin{tabular}{|c|c|c|c|}
\hline \multirow{3}{*}{$\begin{array}{r}\text { Step between the } \\
\text { pass }\left(a_{\mathrm{e}}\right), \mathrm{mm}\end{array}$} & \multirow{2}{*}{\multicolumn{3}{|c|}{$\begin{array}{r}\text { Rotational speed of the spindle } \\
n, \text { rpm }\end{array}$}} \\
\hline & & & \\
\hline & 4000 & 8000 & 12000 \\
\hline 0,04 & 14,58 & 8,33 & 6,25 \\
\hline 0,08 & 7,29 & 4,17 & 3,13 \\
\hline 0.12 & 4,86 & 2,78 & 2,08 \\
\hline
\end{tabular}
line high-speed milling with a half-overlap in the treatment of a flat surface area $\mathrm{S}=25 \mathrm{~cm}^{2}$

It should be noted that since the cutting depth is minimal when processing with the technology of high-speed milling with half-overlapping, it is not rational to consider the volume productivity to evaluate the efficiency of the technology. It is necessary to analyze the productivity of the technology in terms of time required to completely cover the surface by the cutter passes.

To ensure correlation between the calculated time and real time of surface treatment with different combinations of processing modes, it is necessary to take into account the type of material (Young module, hardness), the type of tool (material, coating), type of cooling.

However, for a preliminary evaluation of the application performance of the developed method, the proposed mathematical approach is sufficient.

\section{DISCUSSION}

The development of the technology of manufacturing parts using the technology of highspeed milling with half-overlap requires consideration of a complex of technological solutions from the design of high-precision CADmodels, to their production on modern machining centre modules.

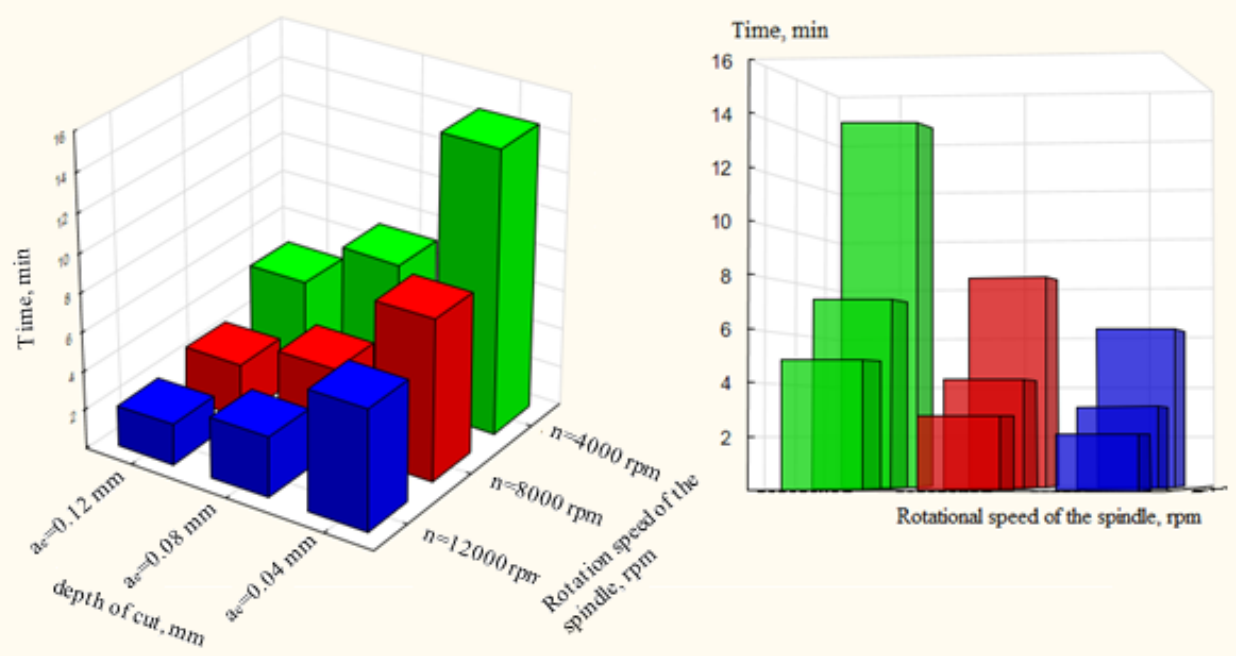

Fig. 4. Dependence of machining time by technology of high-speed milling with half-overlap from the step between the passes and the rotation speed of the spindle 
The choice of a rational route of the technological process of manufacturing parts of any complexity depends on the required quality characteristics, put forward to the working surfaces of the finished product.

In addition, the cost of manufacturing products depends on the choice of the technological process. That is why the application of high-speed milling technology with half-overlap can be recommended at the final stages of engineering products manufacturing. And in order to increase the productivity and efficiency of semi-finished processing, we recommend using Vortextechnology (Fig. 5) (vortex-vibrational technology) developed by Delcam.

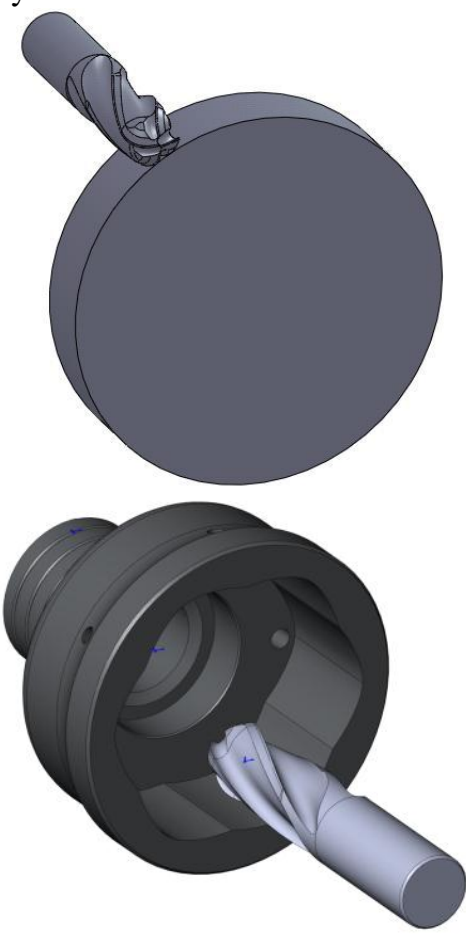

Fig. 5. Milling with Vortex technology

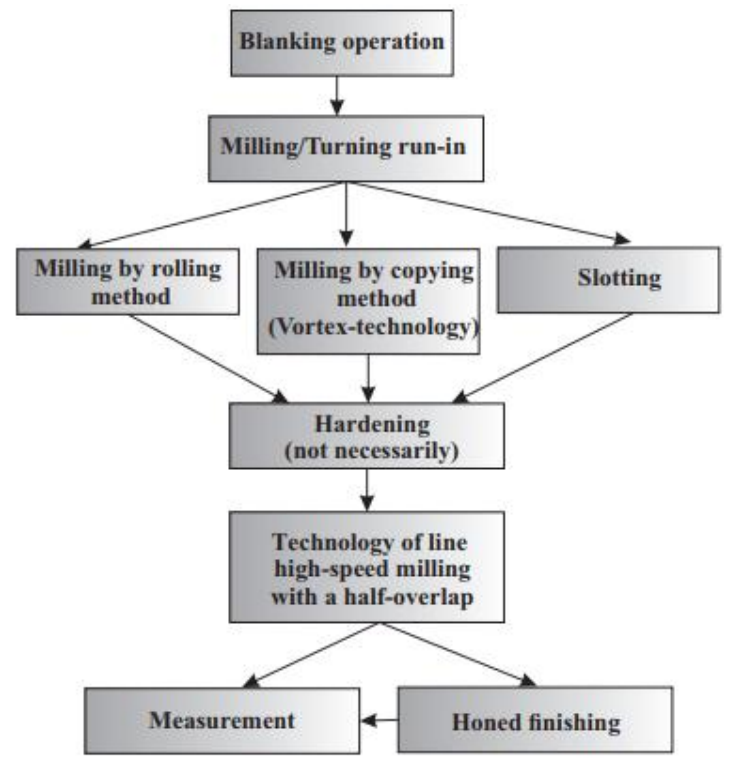

Fig. 6. The recommended route for machining the surfaces of composite parts
Based on the above information and on the knowledge that the use of modern machining centre modules involves the maximum concentration of the processing operations on one equipment, we concluded that the following sequence of actions may be promising when forming complex shape parts (Fig. 6).

\section{CONCLUSIONS}

1. Based on the up-to-date knowledge of the physics of the formation of a qualitative surface, the high-speed milling technology with halfoverlap is proposed. It is assumed that such a method of processing at the final stage of manufacturing products is able to provide a quality of surface part equivalent to the quality after grinding. Such an effect can be explained through an understanding of the work of all components of the complex energy-intensive process of high-speed milling.

2. A methodology for evaluating the processing efficiency of high-speed milling technology with half-overlap has been developed. It is established that the technology of high-speed milling with half-overlap allows to obtain a sufficiently high specific productivity of the processing $\mathrm{Q}=12.5 \div 4.2 \mathrm{~cm}^{2} / \mathrm{min}$ depending on the technological regimes.

3. The received results of theoretical researches are a basis of carrying out of experimental works on comparative tests of technology of high-speed milling with a half overlap and without application of half-overlap. This will allow to determine the level of influence of the deformation component of the cutting process on the roughness and the physical and mechanical properties of the material after cutting.

\section{REFERENCES}

1. Monreal M, Rodriguez Ciro A. Influence of tool path strategy on the cycle time of high-speed milling. Computer-Aided Design. 2003;33:393-401. https://doi.org/10.1016/S0010-4485(02)00060-X

2. Tang Yi. Optimization strategy in end milling process for high speed machining of hardened die/mold steel. Journal of the University of Science and Technology Beijing, Mineral, Metallurgy, Material, 2006; 13(3): 240-243.

https://doi.org/10.1016/S1005-8850(06)60051-0

3. CNC cookbook. be a better CNC'er. Electronic resource. Date of last treatment 08.03.2018 https://www.cnccookbook.com/high-speedmachining-trochoidal-milling-hsm-speeds-and-feeds/

4. Tool path strategies for high speed machining. modern machine shop. Date of last treatment 08.03.2018

https://www.mmsonline.com/articles/tool-pathstrategies-for-high-speed-machining

5. Toh CK. Cutter path strategies in high speed rough milling of hardened steel. Materials and Design 2006; 27(2): 107-114.

https://doi.org/10.1016/j.matdes.2004.09.021 
6. Pena AE, Anania FD, Zapciu M. Research concerning optimum cutting parameters according with tool path strategy for finishing procedures. IOP Conference Series: Materials Science and Engineering. 2015; 95(1): 12-20. https://doi.org/10.1088/1757-899X/95/1/012020

7. Shajari S, Sadeghi MH and Hassanpour H. The influence of tool path strategies on cutting force and surface texture during ball end milling of low curvature convex surfaces. Scientific World Journal 2014; eCollection 2014

https://doi.org/10.1155/2014/374526.

8. Mebrahitom A, Rizuan D, Azmir M. Nassif M. Effect of high-speed milling tool path strategies on the surface roughness of Stavax ESR mold insert machining. IOP Conference Series: Materials Science and Engineering 2016; 114(1): 1-6. https://doi.org/10.1088/1757-899X/114/1/01200

9. Msaddek EB, Bouaziz Z, Dessein G, Baili M Optimization of pocket machining strategy in HSM. The International Journal of Advanced Manufacturing Technology. 2012; 62 (1-4): 69-81. https://doi.org/10.1007/s00170-011-3801-9

10. Dobrotvorskiy SS. Scientific basis of the process of laser cutting of grinding wheels from superhard materials. Abstract of the thesis for the degree of a doctor of technical sciences. Kiev 1996. Ukrainian.

11. Basova EV. Technological support of quality and accuracy of surfaces of parts from hardened chromemolybdenum steels by the method of high-speed milling. Abstract of the thesis for the degree of the candidate of technical sciences. Kharkov 2014.

12. Chiou J, Yuan-Shin L. Five-axis high speed machining of sculptured surfaces by surface-based NURBS path interpolation. Computer-Aided Design and Applications 2007; 4(5): 639-648 https://doi.org/10.1080/16864360.2007.10738498

13. Li K. Fundamentals of automated design engineering system (CAD / CAM / CAE). Piter 2004. Rusian.

14. Piegl L, Tiller W. The NURBS Book, 2nd Edition, Springer-Verlag Berlin Heidelberg, Germany 1997.

15. Chiou, J, Yuan-Shin L. Machining potential field approach to tool path generation for multi-axis sculptured surface machining. Computer-Aided Design 2002; 34(5):357-371. https://doi.org/10.1016/S0010-4485(01)00102-6

Received 2018-04-05

Accepted 2018-07-10

Available online 2018-07-11

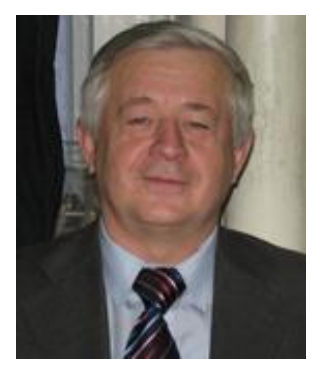

Sergey DOBROTVORSKIY (ORCID 0000-0003-1223-1036), Doctor of technical sciences, Full Professor, Professor at the Department of mechanical engineering and metal-cutting machine tools of the Educational and scientific institute of mechanical engineering and transport of the NTU «KhPI»,

Kharkov, Ukraine.

Head at the "Technopolis Engineering"LLC and "JavaMach cluster".

Main directions of scientific research: physicotechnical processing methods, engineering technology, programming, CAD / CAM / CAE / CAPP systems, Industry 4.0 .

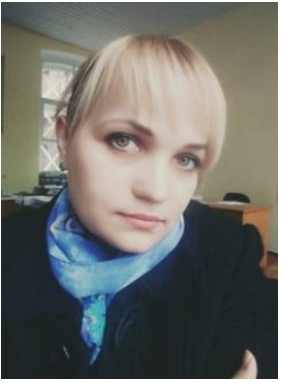

Yevheniia BASOVA (ORCID 0000-0002-8549-4788), PhD, Associate Professor at the Department of technology of mechanical engineering and metal-cutting machine tools of the Educational and scientific institute of mechanical engineering and transport of the NTU «KhPI», Kharkov, Ukraine. Main directions of scientific research: physicotechnical processing methods, engineering technology, programming, CAD / CAM / CAE / CAPP systems, optimization and modelling in mechanical engineering processes for technological prognostication in innovations.

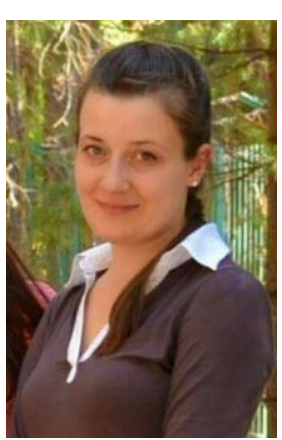

Maryna IVANOVA (ORCID 0000-0002-0848-6805), PhD, Associate Professor at the Department of technology of mechanical engineering and metal-cutting machine tools of the Educational and scientific institute of mechanical engineering and transport of the NTU «KhPI», Kharkov, Ukraine.

Main directions of scientific research: enhancement of functional capabilities and an increase productivity metal-cutting machines; multicriteria optimization of technological processes and systems.

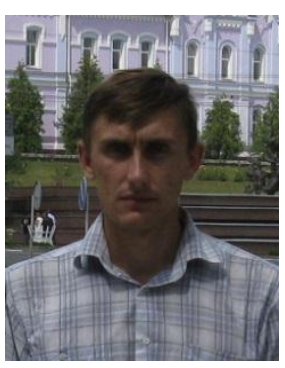

Alexey KOTLIAR (ORCID 0000-0001-7664-0395), $\mathrm{PhD}$, Docent, Associate Professor at the Department of technology of mechanical engineering and metal-cutting machine tools of the Educational and scientific institute of mechanical engineering and transport of the NTU «KhPI», Kharkov, Ukraine. Main directions of scientific research: enhancement of functional capabilities and an increase productivity metal-cutting machines; multi-criteria optimization of technological processes and systems.

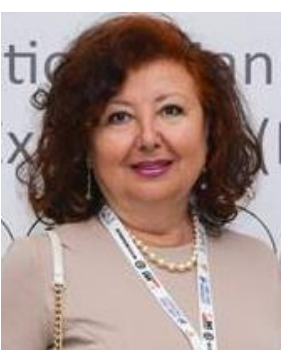

Kharkov, Ukraine.
Main directions of scientific research: physicotechnical processing methods, engineering technology, programming, CAD / CAM / CAE / CAPP systems, Industry 4.0. 